\title{
Influencia de pausa activa en factores de riesgo ergonómicos en trabajadores de limpieza de una universidad privada.
}

Influence of active pause in ergonomic risk factors in cleaning workers of a private university.

\author{
Marilyn Aurora López Sarmiento 1,2,a, María Elena Toribio Mestanza ${ }^{1,3, a}$, Aurora Marlene Giraldo Giraldo 1,a,b,c,d.
}

\section{RESUMEN}

Objetivo: Determinar la influencia de la intervención de pausa activa en factores de riesgo ergonómicos en trabajadores de limpieza. Material y métodos: enfoque cuantitativo, pre experimental que involucró a 75 trabajadores del área de limpieza. Se empleó el instrumento de Ovako Working Analysis System (OWAS) en su versión español para identificar prevalencia de riesgo ergonómico mediante registros fotográficos y un cuestionario para evaluar la intervención de pausa activa antes y después, siendo analizado mediante la prueba Chi cuadrado. Resultados: En el factor individual predominó sexo masculino (54,7\%); el grupo etario de 31 a 45 años (46,7\%); tiempo de permanencia laboral inferior de 5 años desarrollando actividades de limpieza (78,6\%). Los trabajadores presentaron al menos una categoría de riesgo ergonómico inicial. Según el instrumento de OWAS, se encontró un 38,6\% en la categoría de riesgo 3 (efecto de postura dañina sobre el sistema músculo-esquelético). Se identificó que el 73,3\% presentó factores de riesgo ergonómico como molestias músculo esqueléticas en espalda, brazos, piernas antes de la intervención y disminuyeron las molestias a $20 \%$ post intervención. Antes de la intervención el factor de riesgo según postura forzada y movimiento repetitivo en espalda fue de $42,7 \%$ y disminuyó a $12 \%$ post intervención. Se identificó que la influencia de la intervención de pausa activa en los factores de riesgo ergonómicos antes y después fue significancia $(\mathrm{p}<0,01)$. Conclusiones: existe influencia de pausa activa en los factores de riesgo ergonómicos en los resultados antes y después de la intervención.

PALABRAS CLAVE: Pausa de seguridad en la atención a la salud, ergonomía, trabajadores, enfermería, salud ocupacional.

\section{SUMMARY}

Objective: To determine the influence of the intervention of active pause on the ergonomic risk factors of the cleaning workers. Material and Methods: The study was of pre-experimental type, quantitative approach, testing results before and after the intervention. The instrument applied was the Ovako Working Analysis System (OWAS), in its Spanish version, to identify the categories of ergonomic risk through observation of the positions of their work activities with photographic record of each worker, being analyzed by the chi-squared test. Results: Male sex prevailed in the individual factor (54.7\%); the age group from 31 to 45 years old (46.7\%); working time of less than 5 years developing cleaning activities (78.6\%). The workers presented at least one category of initial ergonomic risk according to the OWAS instrument, 38.6\% was found in the risk 3 category (harmful posture effect on the musculoskeletal system). It was identified that $73.3 \%$ presented ergonomic risk factors such as musculoskeletal discomfort in the back, arms, legs before the intervention and decreased discomfort to $20.0 \%$ post intervention. Before the intervention, the risk factor according to forced posture and

\footnotetext{
${ }^{1}$ Facultad de Enfermería, Universidad Peruana Cayetano Heredia. Lima,Perú.

${ }^{2}$ Clínica Internacional - Sede San Borja. Lima, Perú.

${ }^{3}$ Clínica del Dolor. Lima, Perú.

${ }^{\text {a }}$ Licenciada.

${ }^{\mathrm{b}}$ Profesor auxiliar.

${ }^{c}$ Maestro en Gestión del Cuidado en Enfermaría.

${ }^{\mathrm{d}}$ Especialista en Salud Ocupacional.
} 
repetitive movement in the back was $42.7 \%$ and decreased to $12.0 \%$ post intervention. It was identified that the influence of active pause intervention on the ergonomic risk factors before and after was significant $(\mathrm{p}<0.001)$. Conclusions: there is an active pause influence on the ergonomic risk factors in the results before and after the intervention.

KEYWORDS: Time out, ergonomics, workers, nursing, occupational health.

\section{INTRODUCCIÓN}

La Organización Internacional del Trabajo (OIT), refiere que se ha incrementado el reporte de casos de muerte por accidentes o enfermedades relacionadas con el trabajo de 6300 personas a 2.3 millones de muertes por año (1). De esta manera los datos permiten visualizar la problemática existente en el mundo laboral, debido a que existe exposición a riesgos en dicho entorno, aspecto que se puede presentar cuando el puesto no se adapta al trabajador, que se suma a la informalidad que existe en no asumir los riesgos laborales como punto de partida para garantizar la salud de este grupo ocupacional.

En tal sentido la adaptación del puesto de trabajo permite beneficios sobre todo ergonómicos como bien lo define la OIT, considerando que la aplicación de la ergonomía se torna beneficioso en el ambiente laboral; enfatizando en el trabajador condiciones laborales más sanas y seguras; y por otro lado reflejando para el empleador, el incremento de la productividad (2).

De esta manera surgen los factores de riesgo individual, que se caracteriza por quien realiza las tareas como son la edad, género, aptitud física y tiempo laboral. Además, los factores de riesgos ergonómicos son condiciones asociados a las actividades del trabajo consideradas tareas, donde los trabajadores realizan posturas forzadas, y en algunas circunstancias movimientos repetitivos; así como manipulación de cargas que influyen en la aparición de enfermedades músculo esqueléticas, siendo parte de la lista de enfermedades ocupacionales $(2,3)$.

En el Perú, según el Plan Nacional de Seguridad y Salud en el Trabajo 2017 - 2021, se establece como prioridad "La salud ocupacional cuya estrategia es la promoción y prevención en accidentes o enfermedades profesionales"(4), esto se respalda por la ley No 29783 enfocada en cuanto a seguridad y salud laboral; asimismo cabe resaltar que la Norma Básica de Ergonomía y de Procedimiento de Evaluación de Riesgo en el artículo $5^{\circ}$ de la ley $\mathrm{N}^{\circ} 27711$, menciona que el actual entorno precisa de normas preventivas con la finalidad de proteger de riesgos ocupacionales para asegurar la salud integral de los trabajadores, con el propósito de mejorar las condiciones y el medio ambiente de laboral (5).

Por su parte, el puesto laboral del trabajador de limpieza es desarrollar actividades de forma manual, ejerciendo movimientos físicos y posturas como: barrer, fregado de pisos, limpieza de ventanas, limpieza de polvo en diferentes superficies, donde se adopta posturas estáticas, en posición de pie recargando el peso en las piernas, así como la manipulación de los deshechos que por lo general se acumulan en bolsas de basura. Así mismo, requieren manipular baldes de agua, uso de máquina lustradora cuyas acciones de empuje y arrastre es frecuente sometiendo al cuerpo a diversas posturas inadecuadas, obligando adoptar posturas forzadas y encorvamiento excesivo de la espalda.

Ante ello se hace necesario conocer estrategias apropiadas que favorezcan el cuidado de este grupo ocupacional, con la finalidad de fortalecer la promoción y prevención en el ámbito laboral del trabajador de limpieza para el bienestar y la salud, siendo un pilar importante en el desarrollo de una organización ya que es un sector que brinda confort, higiene y comodidad a la población dando un servicio indispensable para garantizar la protección y seguridad con un ambiente saludable en el trabajo.

Respecto al tema se evidencian mayoritariamente investigaciones en el área administrativa, como la investigación de Mosquera B. (6); también la investigación de Vallejo J. (7). Así mismo existen estudios de pausa activa en países como Ecuador en la investigación de Mosquera B. (6) además en Chile con Martínez M. (8). Sin embargo a nivel de Perú no se han identificado estudios en la población de trabajadores de limpieza, a pesar que este es un grupo vulnerable por los factores ergonómicos y el ritmo o frecuencia que emplean en su trabajo.

Es así que, ante la problemática planteada el presente estudio pretende determinar la influencia de la intervención de pausa activa en los trabajadores de limpieza de la Universidad Peruana Cayetano Heredia.

\section{MATERIAL Y MÉTODOS}

El estudio fue de enfoque cuantitativo con diseño de tipo pre experimental, que se llevó a cabo en la Universidad Peruana Cayetano Heredia (UPCH), institución académico - universitaria, ubicado en un distrito urbano de Lima. La población total está conformada por 80 trabajadores, se aplicaron los criterios de inclusión y exclusión en la población; los criterios son: que desempeñen funciones en las instalaciones de la UPCH, sean trabajadores mayores de edad (18 a 60 
años), de ambos sexos, tengan jornadas de trabajo de 8 horas diarias, con un tiempo laboral permanente y continuo mayor a 3 meses y que aceptaron participar de forma voluntaria, es por ello que 75 trabajadores de limpieza cumplieron con los criterios de selección y aceptaron participar voluntariamente.

Se aplicó el cuestionario validado por Gavio M. (10), que consta de 16 preguntas que permiten indagar sobre factores individuales del trabajador y las molestias músculo esquelético en brazo, pierna y espalda, así como la manipulación de carga. Por lo tanto, se aplicó antes y después de la intervención de la pausa activa.

En el estudio, se aplicó el instrumento de OWAS y la encuesta. El instrumento de OWAS se realizó con la observación inicial de las diferentes posturas adoptadas durante el desarrollo de sus tareas según actividades "in situ" en el puesto de trabajo del personal de limpieza, mediante el registro con fotografías de las posturas y movimiento durante una semana dentro de su jornada laboral, de esta manera se identificó la prevalencia de riesgo ergonómico inicial según el instrumentó de OWAS que se aplicó antes de la intervención de pausa activa. Utilizando el software online para la evaluación ergonómica en las diferentes posiciones (9). Cabe resaltar que la intervención de la pausa activa se realizó según el protocolo validado por Acosta $M$. en trabajadores de la Empresa "Línea directa", que se dedica al diseño y producción de calzado y ropa interior y exterior para hombres y mujeres en Medellín, autorizado y revisado por Seguros de Riesgos Profesionales Suramericana S.A ARP Sura (11). Por consiguiente, aplicamos el protocolo en los trabajadores de limpieza con una secuencia de pasos, con un tiempo de duración de 10 a 15 minutos en los turnos mañana y tarde, con una frecuencia de 2 veces por día, para ello se realizó la pausa preparatoria al inicio de sus actividades laborales y la pausa compensatoria durante sus actividades laborales durante 1 mes comprendido desde el 15 de noviembre al 15 de diciembre de 2017.

Para el procedimiento de recolección de datos, se contó con la aprobación del Comité Institucional de Ética de UPCH, y se solicitó la autorización a la División de Servicios Generales y su jefatura de personal de limpieza - UPCH. Se invitó a una reunión a todos los trabajadores y se dio a conocer los objetivos de la investigación haciendo la entrega del consentimiento informado.

Se codificaron los datos recolectados e ingresaron a una base de datos en Microsoft Excel versión 2016, conformada por 75 encuestas aplicadas al número de trabajadores de limpieza.

\section{RESULTADOS}

En el factor individual de los trabajadores de limpieza se observó predominio de sexo masculino (55\%), el grupo etario está en el rango de 31 a 45 años (47\%) y además en el tiempo laboral inferior de 5 años dedicadas a las actividades de limpieza (79\%), según se detalla en la Tabla 1. Como resultado de la prevalencia de riesgo ergonómico inicial según el instrumento de OWAS, todos los trabajadores presentaron al menos un factor de riesgo ergonómico se encontró, el $(38,6 \%)$ en la categoría de riesgo 3 (efecto de postura dañina sobre el sistema músculo-esquelético) siendo el porcentaje más alto, acorde a la Tabla 2 .

Se observa como resultado en la tabla 3 que el $73,3 \%$ presentó factor de riesgo y después se mantuvo en $20 \%$. Además, uno de los factores de riesgo con más relevancia fue según postura forzada y movimiento repetitivo en

Tabla 1. Factor individual del Trabajador de Limpieza que brindan servicios en la Universidad Peruana Cayetano Heredia S.M.P, Noviembre- diciembre 2017.

\begin{tabular}{llcc}
\hline Variables & Categorías & n & \% \\
\hline Sexo & Masculino & 41 & 55,0 \\
& Femenino & 34 & 45,0 \\
Edad & 18 a 30 años & 16 & 21,0 \\
& 31 a 45 años & 35 & 47,0 \\
Tiempo laboral & 46 a 60 años & 24 & 32,0 \\
& De 3 meses a 5 años & 59 & 79,0 \\
& De 5 años 1 mes a 10 años & 9 & 12,0 \\
& De 10 años 1 mes a 15 años & 1 & 1,3 \\
& De 15 años 1 mes a 20 años & 2 & 2,6 \\
& De 20 años 1 mes a más & 4 & 5,3 \\
\hline
\end{tabular}


Tabla 2. Prevalencia de riesgo ergonómico inicial según el instrumento de OWAS, en los Trabajadores de Limpieza que brindan servicios en la Universidad Peruana Cayetano Heredia S.M.P, Noviembre-Diciembre 2017.

\begin{tabular}{clcc}
\hline $\begin{array}{c}\text { Categorías } \\
\text { de riesgo }\end{array}$ & Efectos de posturas & n & $\mathbf{\%}$ \\
\hline 1 & $\begin{array}{l}\text { Postura normal sin efecto dañino en el sistema músculo } \\
\text { esquelético. }\end{array}$ & 10 & 13,3 \\
2 & $\begin{array}{l}\text { Posturas con posibilidades de causar daño al sistema } \\
\text { músculo-esquelético. }\end{array}$ & 23 & 30,6 \\
3 & $\begin{array}{l}\text { Postura con efectos dañinos sobre el sistema músculo- } \\
\text { esquelético. }\end{array}$ & 29 & 38,6 \\
4 & $\begin{array}{l}\text { Manipulación de carga causada por estado postural de } \\
\text { carga con efectos dañinos sobre el sistema músculo- } \\
\text { esquelético. }\end{array}$ & 13 & 17,3 \\
Total & & 75 & 100,0 \\
\hline
\end{tabular}

Tabla 3. Influencia de la intervención de pausa activa en los factores de riesgo ergonómicos antes - después en los Trabajadores de Limpieza que brindan servicios en la Universidad Peruana Cayetano Heredia. S.M.P, noviembre diciembre 2017.

\begin{tabular}{llcccccc}
\hline & \multicolumn{3}{c}{ Antes } & \multicolumn{3}{c}{ Después } \\
\cline { 2 - 8 } & & $\mathbf{n}$ & $\mathbf{9}$ & $\mathbf{n}$ & $\mathbf{p}$ \\
\hline Presentó factores de riesgo (molestias músculos esqueléticas). & $\mathrm{No}$ & 20 & 26,7 & 60 & 80 & $<0.001$ \\
& $\mathrm{Si}$ & 55 & 73,3 & 15 & 20 & \\
Factor de riesgo según postura forzada y movimiento & $\mathrm{No}$ & 43 & 57,3 & 66 & 88 & $<0.001$ \\
repetitivo en espalda. & $\mathrm{Si}$ & 32 & 42,7 & 9 & 12 & \\
Factores de riesgo según manipulación de carga en brazos. & $\mathrm{No}$ & 62 & 82,7 & 72 & 96 & 0.017 \\
& $\mathrm{Si}$ & 13 & 17,3 & 3 & 4 & \\
Factores de riesgo según postura forzada & $\mathrm{N}$ movimiento & $\mathrm{No}$ & 65 & 86,7 & 72 & 96 & 0.082 \\
repetitivo en piernas. & $\mathrm{Si}$ & 10 & 13,3 & 3 & 4 & \\
\hline
\end{tabular}

Chi cuadrado y el valor de $\mathrm{P}=\leq 0,05$

espalda antes un $42,7 \%$ y después disminuyó en $12 \%$. Cabe destacar que la intervención de pausa activa en los factores de riesgo ergonómicos antes y después fue significativa en la prueba $\mathrm{Chi}^{2}=40,74(\mathrm{p}<0,001)$.

\section{DISCUSIÓN}

El estudio fue realizado en una universidad privada, donde se evaluaron a 75 trabajadores dedicados a la limpieza de ambientes, esta investigación se concentra en la actividad productiva y el impacto de riesgo que demanda dichas actividades.

En relación al factor individual del trabajador, siendo predominio el sexo masculino con un $54,7 \%$, el grupo etario de 31 a 45 años con un $46,7 \%$ y con un tiempo de permanecía laboral inferior de 5 años con un 78,6\% desarrollando actividades de limpieza, es un indicador importante porque nos permite explicar que a más edad se va incrementando la permanencia en el ámbito laboral, con una jornada de trabajo continuo de 8 horas diarias; en los hallazgos de los estudios mencionados realizado por Gavio M, donde se evaluó a los trabajadores de limpieza que en su mayoría corresponden a las edades de 36 a 60 años y de 20 a 35 años, en su mayoría del sexo masculino y con mayor tiempo de antigüedad laboral (10), ambos estudios coinciden con los factores individuales de los trabajadores de limpieza. Según los resultados encontrados entre la edad y tiempo laboral, puede ser un indicador que influye en el grupo de estudio, siendo susceptible de padecer factores de riesgo ergonómicos porque con el paso de los años el trabajador se expone frecuentemente a condiciones del trabajo donde realizan posturas inadecuadas de manera continua, que exigen movimientos manuales repetitivos e incrementando el riesgo en su salud donde puede causar daños al sistema músculo esquelético. 
Cabe resaltar que la prevalencia de riesgo ergonómico inicial según el instrumento de OWAS; se encontró que los 75 trabajadores estudiados presentaron algún tipo de las 4 categorías de riesgos ergonómicos inicial mediante este instrumento, por lo cual ocasionara un efecto dañino sobre el sistema músculo-esqueléticos.

Con respecto a los factores de riesgo ergonómicos antes de la intervención de la pausa activa, se encontró un $42,7 \%$ con factor de riesgo ergonómico que presenta postura forzada y movimiento repetitivo en espalda un $17,3 \%$ en factores de riesgo según manipulación de carga en brazos y un $13,3 \%$ en el factor de riesgo según postura forzada y movimiento repetitivo en piernas. Cuyos hallazgos que coinciden con el estudio de Corcuera M; donde la postura forzada se localizó en espalda con un $85,3 \%$ (12). Cabe mencionar que se encontró similitud en el estudio de Gavio M, donde presentan un porcentaje de $88,6 \%$ donde los trabajadores presentan molestias en espalda y en menor porcentaje un $11,4 \%$ en brazos y piernas (10). Otro estudio que coincide es Bonilla, J. donde se encontró en los trabajadores de limpieza que manifestaron tener el $64 \%$ de molestias en la espalda y en el brazo derecho e izquierdo un 38\% y pierna derecha e izquierda un 45\% (13). Estos 3 estudios reafirman los resultados donde resalta en porcentaje elevado en el factor de riesgo ergonómico según molestias en espalda, el cual establece una relación con los resultados de la investigación.

A consecuencia se deduce que al adoptar posturas estáticas con manipulación de peso estimado por encima del indicado con acciones de empuje y arrastre frecuente, se somete al cuerpo a diversas posturas forzadas y encorvamiento excesivo de espalda que condiciona a que el cuerpo adopte posturas viciosas sobre todo cuando es continua y con cierta frecuencia sin una pausa establecida. De esta manera se determinó la influencia de la intervención de la pausa activa en los factores de riesgo ergonómicos antes y después. Donde los factores de riesgo según postura forzada y movimiento repetitivo en espalda antes un $42,7 \%$ y después un $12 \%$, factores de riesgo según manipulación de carga en brazos antes un $17,3 \%$ y después un $4 \%$ y factores de riesgo según postura forzada y movimiento repetitivo en piernas antes un $13,3 \%$ y después un 4\%. Así mismo en la investigación de Corcuera M. en su Pos test después de la intervención de la pausa activa se evidencia que las molestias en espalda antes con un $85,3 \%$ y después disminuyeron con un $44 \%$ (12). Se evidencia que ambas investigaciones existen una diferencia significativa al comparar los resultados antes y después de la intervención de la pausa activa.

De esta forma se deduce que la similitud con otros estudio permite presentar evidencia que respalda la intervención de la pausa activa como una medida preventiva y que nuestro resultados estadísticamente significativos cuando se compara un antes y después de la intervención de la pausa activa como bien resalta Olivera J. donde manifiesta que la pausa activa beneficia en el incremento de la circulación sobre todo en estructuras musculares, por lo que se espera mejorar la oxigenación muscular, de tendones y a fin de disminuir el acúmulo de ácido láctico. Por su parte, para mejorar la movilidad articular y por ende la flexibilidad muscular que favorece la postura y disminuye los accidentes laborales, mejorando así la capacidad de concentrarse durante la actividad en labora y promoviendo la integración social (14).

Cabe mencionar que la intervención de la pausa activa, tras su aplicación ha demostrado influenciar en la mejora de la postura principalmente en espalda, asimismo brazos y piernas partiendo del diagnóstico inicial de los factores de riesgo ergonómicos empleando el instrumento de OWAS, permitiendo identificar en tiempo real según la tarea de los trabajadores las categorías de riesgo inicial.

\section{CONCLUSIONES}

La intervención de pausa activa influye en los factores de riesgo ergonómicos, debido a que se identifican cambios comparativos entre el antes y después de la intervención.

Por su parte, los trabajadores de limpieza en la categoría de riesgo ergonómico inicial presentaron alto porcentaje de riesgo, evidenciado por puntaje 3; posturas con posibilidad de causar daño sobre el sistema musculo esquelético; predominando antes de la pausa activa la postura forzada y movimiento repetitivo en espalda.

En cuanto a los factores de riesgo ergonómico post intervención con pausa activa fue en dos momentos pausa preparatoria (antes de la jornada laboral) y pausa compensatoria (durante la jornada laboral). Se evidenció una disminución en cada factor de riesgo ergonómico.

\section{Correspondencia:}

Marilyn Aurora López Sarmiento.

Correo electrónico: Marilyn.lopez.s@upch.pe

\section{REFRENCIAS BIBLIOGRÁFICAS}

1. Organización Internacional del Trabajo. Seguridad y salud en el trabajo. Washington: Organización Internacional del Trabajo. (Citado el 26 de diciembre del 2017). Disponible en: https://www.ilo.org/global/ topics/safety-and-health-at-work/lang--es/index.htm

2. Organización Internacional del Trabajo. La Salud y la Seguridad en el Trabajo: La Ergonomía. Washington: Organización Internacional del Trabajo; 
2016. (Citado el 26 de diciembre del 2017). Disponible en: http://white.lim.ilo.org/spanish/260 ameri/oitreg/activid/proyectos/actrav/proyectos/pdf/ ergonomia.pdf

3. Confederación de Organizaciones Empresariales de Murcia (CROEM), Instituto de Seguridad y Salud Laboral de la Región de Murcia (ISSL). Prevención de riesgo ergonómico. España: Confederación de Organizaciones Empresariales de Murcia; 2017. (Citado el 26 de diciembre del 2017). Disponible en: http://www.croem.es/prevergo/formativo/1.pdf

4. Ministerio de Salud. Decreto Supremo que aprueba el Plan Nacional de Seguridad y Salud en el Trabajo 2017 - 2021Peru; Ministerio de Salud; 2017. (Citado el 26 de diciembre del 2017). Disponible en: elpe ruano.pe/normaslegales/decreto-supremo-queaprueba-el-plan-nacional-de-seguridad-y-decretosupremo-n-005-2017-tr-1509246-3/

5. Villasante J. Norma Básica de Ergonomía y de Procedimiento de Evaluación de Riesgo Disergonómico. Lima: MTC; 2016. (Citado el 26 de diciembre del 2017). Disponible en: https://www. mtc.gob.pe/nosotros/seguridadysalud/documentos/ RM\%203752008\%20TR\%20\%20Norma\%20 B\%C3\%A1sica\%20de\%20Ergonom\%C3\%ADa.pdf

6. Mosquera B. Las pausas activas laborales y su incidencia en el desempeño laboral de los trabajadores del área de ahorro y crédito de la cooperativa educadores de Tungurahua de la ciudad de Ambato. Ambato:Universidad Tecnica de Ambato;2016. (Citado el 26 de diciembre del 2017). Disponible en: http://repositorio.uta.edu.ec/jspui/ handle/123456789/22910

7. Vallejo J. Evaluación de los niveles de actividad física en el personal de oficina administrativo de la PUCE, para la elaboración de una propuesta de pausa laboral activa como estrategia de atención primaria en salud 2013. Quito: Pontificia Universidad Católica del Ecuador. (Citado el 26 de diciembre del 2017). Disponible en:http://repositorio.puce.edu.ec/handle/ 22000/5988
8. Martínez M. efecto de las pausa activas en el dolor musculo esquelético en trabajadoras dePacking.. Chile: Universidad de Chile; 2014. (Citado el 26 de diciembre del 2017). Disponible en:http://bibliodigital. saludpublica.uchile.cl:8080/dspace/bitstream/ 123456789/439/1TESIS+MARTA+MARTINEZ+M ALDONADO+.pdf

9. Mas D. Evaluación postural mediante el método OWAS. Ergonautas/ergonomía ocupacional y prevención de riesgos laborales. Valencia, España: Ergonautas. (Citado el 26 de diciembre del 2017). Disponible en: https://www.ergonautas.upv.es/

10. Gavio M. Implicancias de la pausa laboral en empleados activa laboral en empleados de la ciudad del rioja 2014. Mexicana de Medicina Física y Rehabilitación 2006; 18: 38.

11. Acosta M. Protocolo para la intervención del programa de pausas activas. Colombia: RIDSSO; 2013.(Citado el 26 de diciembre del 2017). Disponible en: www.ridsso.com/documentos/muro/207_141696 7597_547535ad912fa.doc

12. Corcuera M. Eficacia de la implementación de un programa de pausas activas en la disminución de síntomas músculo-esqueléticos en trabajadores administrativos de una empresa de servicios de saneamiento. Lima, Perú: Universidad Cesar Vallejo; 2016.

13. Bonilla J. Riesgo ergonómico que afectan al personal de limpieza de la Universidad nacional autónoma de honduras. Nicaragua: Universidad Nacional Autónoma de Nicaragua;2013. (Citado el 26 de diciembre del 2017). Disponible en: http://repositorio. unan.edu.ni/7622/1/t732.pdf

14. Olivera J. Bienestar Fisioterapia Personalizada pausa activa, cuando trabajar hace bien para la salud. Montevideo: BlueCross \& BlueShield de Uruguay; 2016. (Citado el 26 de diciembre del 2017). Disponible en: https://www.bcbsu.com.uy/bcbsu/ archivos/nota_pausaActiva.pdf 